\title{
Four-dimensional Gravity from Singular Spaces
}

\author{
Ph. Brax \\ Service de Physique Théorique \\ CEA-Saclay \\ F-91191, Gif/Yvette cedex, France \\ C. van de Bruck, A. C. Davis, C. S. Rhodes \\ Department of Applied Mathematics and Theoretical Physics \\ Centre for Mathematical Sciences \\ Wilberforce Road, University of Cambridge \\ CB3 0WA, United Kingdom
}

July 29, 2013

\begin{abstract}
The modification to four-dimensional Einstein gravity at low energy in two brane models is investigated within supergravity in singular spaces. Using perturbation theory around a static BPS background, we study the effective four-dimensional gravitational theory, a scalar-tensor theory, and derive the Brans-Dicke parameter when matter is present on the positive tension brane only. We show there is an attractor mechanism towards general relativity in the matter dominated era. The dynamics of the interbrane distance are discussed. Finally, when matter lives on both branes, we find that there is a violation of the equivalence principle whose magnitude is governed by the warping of the extra dimension.
\end{abstract}

DAMTP-2002-25, t02/009

\section{Introduction}

The notion that our Universe may be a hypersurface within a higher-dimensional space-time is not a new one [1, 2, 3], but recently interest in this class of theory of the Universe has been rekindled by the development of simple models, notably by Randall and Sundrum [4] and [5]. These models admit a rich set of developments and extensions. In brane world theories the standard model fields are usually confined on our (visible) brane, while allowing gravity to propagate throughout the entire space-time. Recovering a four-dimensional theory of gravity that is compatible with present-day observations is one of the main constraints to be imposed on brane-world models. Such an analysis has been carried out in the RS framework with an explicit radion stabilization mechanism in $\|$. 
One possible extension to the RS set-up is the inclusion of matter fields in the (extradimensional) bulk. In particular it is of interest to study the dynamics of bulk scalar fields as these fields appear naturally in models motivated from M-theory [7], see $\|8\|-\|21\|$. Recently the issue of retrieving gravity at low energy for models with a single bulk scalar field has been analyzed in [22]. It has been found that four dimensional gravity is generically recovered provided that the models do not have a super-gravitational origin円.

In this paper, we discuss the effect of including a scalar field in the bulk, for certain super-gravitational brane-world models [8, 9, 10, 11], on the effective four-dimensional theory of gravity. To examine this, we build on the results of a companion paper [12 in which we studied the dynamics of linear perturbations of the radion (i.e. fluctuations of the brane distance) and the bulk scalar field. We compute the metric perturbation due to matter on the brane, and compare the low-energy limit to the Brans-Dicke theories; see |23.

We begin by summarizing the relevant results from our companion paper in section 2 and add matter sources on the branes; we then, in section 3, compute the Brans-Dicke parameter that would be observed on the positive-tension brane in the low-energy limit, and show that general relativity is an attractor in rather general cases. We draw our conclusions in section 4 .

\section{Brane World Setup: Background and Perturbations}

\subsection{The Background Configurations}

We are interested in brane world models with a bulk scalar field. In addition, we have two branes in our setup: one (the visible brane) is located at $z=0$, whereas the other (hidden) brane is located at $z=r_{c}$. We consider a bulk action

$$
S_{\mathrm{bulk}}=\frac{1}{2 \kappa_{5}^{2}} \int d^{5} x \sqrt{-g_{5}}\left(R-\frac{3}{4}\left((\partial \phi)^{2}+U\right)\right)
$$

with boundary terms

$$
S_{\text {brane }}=-\frac{3}{2 \kappa_{5}^{2}} \int d^{5} x \sqrt{-g_{5}}\left(\delta(z)-\delta\left(z-r_{c}\right)\right) U_{B}
$$

where $U_{B}$ is the superpotential related to the bulk potential $U$ by

$$
U=\left(\frac{\partial U_{B}}{\partial \phi}\right)^{2}-U_{B}^{2}
$$

Background solutions to the equations of motion are described by the BPS equations

$$
\frac{a^{\prime}}{a}=-\frac{U_{B}}{4}, \phi^{\prime}=\frac{\partial U_{B}}{\partial \phi}
$$

where ${ }^{\prime}=d / d z$, for a metric of the form

$$
d s^{2}=d z^{2}+a^{2}(z) \eta_{\mu \nu} d x^{\mu} d x^{\nu}
$$

${ }^{1}$ More specifically, the authors of [22] assume that the relation $U=U_{B}^{\prime 2}-U_{B}^{2}$ between the brane and the bulk potentials is violated. This relation holds explicitly for the models that we consider. 
We will particularly focus on the case where

$$
U_{B}=4 k e^{\alpha \phi}
$$

with $\alpha=1 / \sqrt{3},-1 / \sqrt{12}$; these values correspond to supergravity in singular spaces $\|8\|$. In those cases the solution reads

$$
a(z)=\left(\frac{1-4 k \alpha^{2}\left(z-z_{0}\right)}{1+4 k \alpha^{2} z_{0}}\right)^{\frac{1}{4 \alpha^{2}}}
$$

where the positive tension brane sits at $z=0$. In the $\alpha \rightarrow 0$ limit we retrieve the AdS profile

$$
a(z)=e^{-k z}
$$

Notice that in that case the scalar field decouples altogether, as $U_{B}$ and hence $U$ are constant.

\section{$2.2 \quad$ Fluctuations}

Our aim is to analyse the influence of the matter localized on the brane. We thus include matter on the boundary branes which will be treated as perturbations. We will further assume that the matter does not couple to the bulk scalar field, so that, in particular, the junction conditions for the bulk field are not modified. As a consequence we are able to build on the analysis performed in $\| 12$.

We consider a perturbed bulk metric

$$
d s^{2}=d z^{2}+a^{2}(z) d x^{\mu} d x_{\mu}+h_{\mu \nu} d x^{\mu} d x^{\nu}
$$

in Gaussian normal coordinates. The dynamics of the scalar field fluctuation is determined by the Klein-Gordon equation

$$
\square \delta \phi+\frac{1}{2} h^{\prime} \phi^{\prime}=\frac{1}{2} \frac{\partial^{2} U_{B}}{\partial \phi^{2}} \delta \phi
$$

where $^{\prime}=d / d z$ and $h$ is the trace of $h_{\mu \nu}$. The zero-mode solution to this equation can be obtained as

$$
\delta \phi=\frac{\partial U_{B}}{\partial \phi} \hat{\phi}\left(x^{\mu}\right)
$$

and $h=0$, which satisfies the boundary conditions at both branes for any massless $\hat{\phi}\left(x^{\mu}\right)$.

Similarly one can write the Einstein equations in the bulk

$$
\square h_{a b}+\frac{U_{B}^{2}}{8} h_{a b}-D_{\{a} D_{c} h_{b\}}^{c}=\frac{3}{4} \partial_{\{a} \delta \phi \partial_{b\}} \phi_{0}
$$

where we have extended $h_{\mu \nu}$ to five dimensions with $h_{55}=h_{\mu 5}=0$. The (55) component of the gravitational equation leads to $\partial_{z} \delta \phi=0$. This implies that $\delta \phi$ is a function of $x^{\mu}$ only and therefore

$$
\delta \phi=0
$$

So the scalar field perturbation vanishes altogether. Now the $(\mu 5)$ equation leads to

$$
D_{\mu} h^{\mu \nu}=0
$$

In summary, we find that the scalar field does not play a rôle in the low energy dynamics and that the metric perturbation is transverse and traceless. 


\subsubsection{Adding matter sources on the branes}

The effect of adding matter on the branes is to induce a bending of the brane. This leads to a shift in the position of both branes which sit at

$$
z_{+}=\xi^{+}, z_{-}=r_{c}+\xi^{-}
$$

One can perform local changes of coordinates around both branes which preserve the Gaussian normal coordinates and shift them to the origin and $r_{c}$. After such changes of coordinates the metric is

$$
h_{\mu \nu}^{+}=h_{\mu \nu}-\frac{U_{B}}{2} a^{2}(z) \xi^{+} \eta_{\mu \nu}-2 a^{2}(z) \int_{0}^{z} \frac{d y}{a^{2}(y)} \partial_{\mu} \partial_{\nu} \xi^{+}
$$

in the vicinity of the origin and

$$
h_{\mu \nu}^{-}=h_{\mu \nu}-\frac{U_{B}}{2} a^{2}(z) \xi^{-} \eta_{\mu \nu}-2 a^{2}(z) \int_{r_{c}}^{z} \frac{d y}{a^{2}(y)} \partial_{\mu} \partial_{\nu} \xi^{-}
$$

in the vicinity of the second brane.

We can now study these perturbations. To do so we come back to the gravitational equation; it is convenient to work in the conformal gauge, defining

$$
d u=\frac{d z}{a}
$$

and

$$
h_{\mu \nu}=\sqrt{a} \tilde{h}_{\mu \nu}
$$

We denote by $u_{+}$and $u_{-}$the location of both branes in the conformal coordinate system. The gravitational equations then read

$$
-\frac{d^{2} \tilde{h}_{\mu \nu}}{d u^{2}}+\frac{1}{a^{3 / 2}} \frac{d^{2} a^{3 / 2}}{d u^{2}} \tilde{h}_{\mu \nu}=\square^{(4)} \tilde{h}_{\mu \nu}
$$

or

$$
\left[Q^{\dagger} Q-\square^{(4)}\right] \tilde{h}_{\mu \nu}=0
$$

with

$$
Q=-\frac{d}{d u}+\frac{d \ln a^{3 / 2}}{d u}, Q^{\dagger}=\frac{d}{d u}+\frac{d \ln a^{3 / 2}}{d u}, \square^{(4)}=\eta^{\mu \nu} \partial_{\mu} \partial_{\nu}
$$

The boundary conditions are now

$$
\left.\partial_{z} h_{\mu \nu}\right|_{u_{ \pm}}+\left.\frac{U_{B}}{2} h_{\mu \nu}\right|_{u_{ \pm}}=-\kappa_{5}^{2} \Sigma_{\mu \nu}^{ \pm}
$$

where

$$
\Sigma_{\mu \nu}^{ \pm}=-\frac{2}{\kappa_{5}^{2}} \partial_{\mu} \partial_{\nu} \xi^{ \pm}+\left(T_{\mu \nu}^{ \pm}-\frac{1}{3} T^{ \pm} g_{\mu \nu}^{ \pm}\right)
$$

and $T_{\mu \nu}^{ \pm}$is the matter energy-momentum tensor on both branes while $g_{\mu \nu}^{ \pm}$is the induced metric.

The equations of motion can be conveniently written as

$$
Q^{\dagger} Q h_{\mu \nu}-\square^{(4)} \tilde{h}_{\mu \nu}=\sum_{\sigma= \pm}\left(\frac{3}{4} U_{B} h_{\mu \nu}^{\sigma}+2 \kappa_{5}^{2} \sqrt{a} \Sigma_{\mu \nu}^{\sigma}\right) \delta\left(u-u_{\sigma}\right)
$$


The brane equation of motion determining the brane bending is obtained by taking the trace of the previous equation. Using the tracelessness of $h_{\mu \nu}$ we conclude that

$$
\square^{(4)} \xi^{ \pm}=-\frac{\kappa_{5}^{2}}{6} a_{ \pm}^{2} T^{ \pm}
$$

where $a_{ \pm}=a\left(u_{ \pm}\right)$.

Let us decompose into Kaluza-Klein modes

$$
\square^{(4)} h_{\mu \nu}=-k^{2} h_{\mu \nu}
$$

It is convenient to define the eigenstates of the positive operator $Q^{\dagger} Q$

$$
Q^{\dagger} Q \psi_{\lambda}=\lambda^{2} \psi_{\lambda}
$$

Consider now the Green's function $G_{k^{2}}$ of the operator

$$
H_{k^{2}}=Q^{\dagger} Q+k^{2}-\frac{3}{4} U_{B}\left(\delta\left(u-u_{+}\right)+\delta\left(u-u_{-}\right)\right)
$$

defined by

$$
G_{k^{2}}\left(u, u^{\prime}\right)=\sum_{\lambda} \frac{\psi_{\lambda}(u) \psi_{\lambda}\left(u^{\prime}\right)}{\lambda^{2}+k^{2}+i \epsilon}
$$

where each mode is normalized. The only mode with $\lambda=0$ is proportional to $a^{\frac{3}{2}}$, as it is the only zero mode to satisfy the boundary conditions imposed by the delta functions. Moreover the spectrum is discrete due to the two boundary conditions.

We can now write the metric perturbation due to matter on both branes

$$
h_{\mu \nu}(u, x)=2 \kappa_{5}^{2} \sum_{\sigma= \pm} \int d^{4} x^{\prime} G\left(x, u ; x^{\prime}, u_{\sigma}\right) a_{\sigma}^{1 / 2} a^{1 / 2}(u) \Sigma_{\mu \nu}^{\sigma}\left(x^{\prime}\right)
$$

where

$$
G\left(x, u ; x^{\prime}, u^{\prime}\right)=\int \frac{d^{4} k}{(2 \pi)^{4}} e^{i k \cdot\left(x-x^{\prime}\right)} G_{k^{2}}\left(u, u^{\prime}\right)
$$

This provides the solution to the Einstein equations in the bulk due to the brane bending.

\section{Gravity on the visible brane: the zero mode trunca- tion}

We concentrate first on the case where no matter is present on the second brane and study gravity on the first brane. In the low energy limit we neglect the contribution due to the Kaluza-Klein modes and retain the zero mode only. In this case the metric perturbation on the brane is

$$
h_{\mu \nu}=-\frac{\kappa_{5}^{2}}{\int_{0}^{r_{c}} d z a^{2}} \frac{1}{\square^{(4)}}\left(T_{\mu \nu}^{+}-\frac{1}{3} T^{+} \eta_{\mu \nu}\right)-\left.\frac{U_{B}}{2}\right|_{0} \xi^{+} \eta_{\mu \nu},
$$

where we have normalized $a_{+}=1$ and used a four dimensional gauge transformation to get rid of the second derivative terms. This leads to

$$
\square^{(4)} h_{\mu \nu}=-\frac{\kappa_{5}^{2}}{\int_{0}^{r_{c}} d z a^{2}}\left(T_{\mu \nu}^{+}-\frac{1}{3} T^{+} \eta_{\mu \nu}\right)+\left.\frac{U_{B}}{12}\right|_{0} \kappa_{5}^{2} T^{+} \eta_{\mu \nu},
$$


Corresponding to the Brans-Dicke expression

$$
\square^{(4)} h_{\mu \nu}=-\frac{16 \pi G_{N}}{\Phi}\left(T_{\mu \nu}^{+}-\frac{1}{2} T^{+} \eta_{\mu \nu}\right)-\frac{8 \pi G_{N}}{\Phi(3+2 \omega(\Phi))} T^{+} \eta_{\mu \nu},
$$

where $\Phi$ is the Brans-Dicke field and $\omega(\Phi)$ the Brans-Dicke parameter. We identify

$$
\kappa_{5}^{2}=16 \pi G_{N} \int_{0}^{r_{\infty}} \frac{a^{2}}{a_{+}^{2}} d z
$$

where $r_{\infty}$ is the maximal range of the extra dimension, i.e. infinity for the Randall-Sundrum scenario and the singularity when a bulk scalar field is present. Next we identify

$$
\Phi=\frac{\int_{0}^{r_{c}} a^{2} d z}{\int_{0}^{r_{\infty}} a^{2} d z}
$$

which varies between zero and one. Finally we obtain the Brans-Dicke parameter

$$
\omega(\Phi)=\frac{3}{2}\left(-1+\frac{1}{1-\left.\Phi \frac{U_{B}}{2}\right|_{0} \int_{0}^{r_{\infty}} \frac{a^{2}}{a_{+}^{2}} d z}\right)
$$

We can now consider the specific exponential models, with $U_{B}=4 k e^{\alpha \phi}$. This leads to

$$
\Phi=1-\left(1-\frac{r_{c}}{r_{\infty}}\right)^{1+\frac{1}{2 \alpha^{2}}}
$$

where $r_{\infty}=z_{0}+1 / 4 k \alpha^{2}$. Similarly for the Brans-Dicke parameter

$$
\omega(\Phi)=\frac{3}{2}\left(-1+\frac{1}{1-\Phi \frac{1+4 k \alpha^{2} z_{0}}{1+2 \alpha^{2}}}\right) .
$$

In the $\alpha \rightarrow 0$ limit we retrieve that

$$
\Phi=1-e^{-2 k r_{c}}
$$

and

$$
\omega(\Phi)=\frac{3}{2}\left(-1+e^{2 k r_{c}}\right)
$$

in agreement with [24].

Let us finally comment on the well known attractor-mechanism for scalar tensor theories in the context of the theory discussed so far. Damour and Nordtvedt 25 found that in the case for Brans-Dicke theory the dynamics of the scalar field evolves during the matter dominated epoch such that the Brans-Dicke parameter grows rapidly and thus that Einstein gravity is a cosmological attractor. It is easy to verify that this is the case in the theory above:

First, it is convenient to go to the Einstein frame where $g_{\mu \nu}=g_{E, \mu \nu} / \Phi$. We then define a canonically normalized field $\sigma$ by $\partial a / \partial \sigma=\beta(\sigma)$ where $a(\sigma)=-\ln (\Phi) / 2$ and $\beta^{2}(\sigma)=1 /(3+2 \omega(\Phi))$, and find that

$$
\beta^{2}(\sigma)=1-\frac{\Phi}{\Phi_{0}}
$$


where $\Phi_{0}=\left(1+2 \alpha^{2}\right) /\left(1+4 \alpha^{2} k z_{0}\right)$. This leads to

$$
\sigma-\sigma_{0}=\frac{1}{2} \ln \left(\frac{1+\beta}{1-\beta}\right)
$$

Notice that $\beta \rightarrow 0$ and $\sigma \rightarrow \sigma_{0}$ as $\Phi \rightarrow \Phi_{0}$. The field $\sigma$ is attracted towards value its value at $\beta=0$, i.e. $\sigma$ is attracted towards $\sigma_{0}$. The minimum $\sigma=\sigma_{0}$ can only been reached when $\Phi_{0} \leq 1$, i.e. for $z_{0} \geq 1 / 2 k$. In that case the field $\sigma$ is attracted towards the point where $\omega(\Phi)=+\infty$, allowing us to recover Einstein gravity.

In the opposite situation $\left(\Phi_{0}>1\right)$ the Brans-Dicke parameter can only reach a maximal value

$$
\omega_{\max }=\frac{3}{2} \frac{1+4 \alpha^{2} k z_{0}}{2 \alpha^{2}\left(1-2 k z_{0}\right)}
$$

which, since $\Phi_{0}>1$ implies that $2 k z_{0}<1$, is in general not large enough to escape the experimental bounds on the Brans-Dicke parameter (and tends to $\frac{3}{4 \alpha^{2}}$ in the limit as $\left.2 k z_{0} \rightarrow 0\right)$.

In conclusion, we have found that the Brans-Dicke parameter converges towards infinity as soon as the distances between the branes and the naked singularity are large enough. This should be the case for a realistic cosmology, because we expect both branes to expand. Thus, they will move away from the singularity so that the attractor mechanism can operate.

Let us finally discuss the case where matter is also on the second brane and study its effect on the gravitational field as seen on the first brane. It is to be expected that the leading effect will be due to the propagation of gravitons in the bulk, hence suppressed by

the warping of the bulk. Following the same method as in the previous calculation we find that

$$
\frac{1}{a_{+}^{2}} \square^{(4)} h_{\mu \nu}=-\frac{16 \pi G_{N}}{\Phi}\left(T_{\mu \nu}-\frac{1}{3}\left(T_{\rho \lambda} \eta^{\rho \lambda}\right) \eta_{\mu \nu}\right)+\left.\frac{U_{B}}{12}\right|_{0}\left(T_{\rho \lambda}^{+} \eta^{\rho \lambda}\right) \kappa_{5}^{2} \eta_{\mu \nu},
$$

where the total energy momentum tensor is

$$
T_{\mu \nu}=T_{\mu \nu}^{+}+\frac{a_{-}^{2}}{a_{+}^{2}} T_{\mu \nu}^{-}
$$

This can be recast into the form

$$
\begin{aligned}
\frac{1}{a_{+}^{2}} \square^{(4)} h_{\mu \nu} & =-\frac{16 \pi G_{N}}{\Phi}\left(T_{\mu \nu}-\frac{1}{2}\left(T_{\rho \lambda} \eta^{\rho \lambda}\right) \eta_{\mu \nu}\right)-\frac{8 \pi G_{N}}{\Phi(3+2 \omega(\Phi))}\left(T_{\rho \lambda} \eta^{\rho \lambda}\right) \eta_{\mu \nu} \\
& -\frac{k}{3} \frac{a_{-}^{2}}{a_{+}^{2}} \kappa_{5}^{2}\left(\eta^{\rho \lambda} T_{\rho \lambda}^{-}\right) \eta_{\mu \nu}
\end{aligned}
$$

where $G_{N}, \Phi$ and $\omega(\Phi)$ were defined above. Notice that there is a direct coupling to matter on the second brane which does not follow from Brans-Dicke theory. In particular this implies that gravity does not couple universally to matter, i.e this leads to a violation of the equivalence principle whose strength is proportional to the warping factor $a_{-}^{2} / a_{+}^{2}$.

\section{Conclusions}

We have presented an analysis of the effective four-dimensional gravity at the positivetension brane for a brane-world model with bulk scalar field. When the BPS conditions (4) 
are imposed on the system, generating a static supergravity background, we find that the effective low-energy theory is a Brans-Dicke theory. We have briefly discussed the attractor mechanism which attracts the theory towards general relativity during a matter dominated epoch. We have found that the conditions for this to happen can easily be fulfilled, as long as the singularity is far away from both branes.

When matter is present on both branes, we have found that both matter types couple differently to gravity. Thus, we expect a violation of the equivalence principle for matter which lives on the shadow brane. This violation, however, is suppressed by a factor, which can be very small, when the branes are far apart. We expect very interesting consequences for cosmology from these considerations, which will be investigated in future work.

Acknowledgements This work was supported by PPARC (A.C.D., C.S.R. and C.v.d.B.), Jesus College, Cambridge (C.S.R.), the Deutsche Forschungsgemeinschaft (C.v.d.B.), a CNRS-Royal Society exchange grant for collaborative research and the European network (RTN), HPRN-CT-2000-00148 and PRN-CT-2000-00148.

\section{References}

[1] K. Akama, An Early Proposal of "Brane World", Lect. Notes. Phys 176 (1982) 267-271, hep-th/0001113.

[2] V. A. Rubakov and M. E. Shaposnikov, Do we live inside a domain wall?, Phys. Lett. B125 (1983) 136.

[3] M. Visser, An exotic class of Kaluza-Klein models, Phys. Lett. B159 (1985) 22, hep-th/9910093.

[4] L. Randall and R. Sundrum, A Large Mass Hierarchy from a Small Extra Dimension, Phys. Rev. Lett. 83 (1999) 3370-3373, hep-th/9905221.

[5] L. Randall and R. Sundrum, An Alternative to Compactification, Phys. Rev. Lett. 83 (1999) 4690-4693, hep-th/9906064.

[6] X. Montes and T. Tanaka, Gravity in the Brane World for Two-Branes Model with Stabilized Modulus, Nucl. Phys. B582 (2000) 259, hep-th/0001092.

[7] A. Lukas, B. Ovrut, K. S. Stelle, and D. Waldram, The Universe as a Domain Wall, Phys. Rev. D59 (1999) 086001, hep-th/9803235.

[8] P. Brax and A.-C. Davis, Cosmological Solutions of Supergravity in Singular Spaces, Phys. Lett. B497 (2001) 289-295, hep-th/0011045.

[9] P. Brax and A.-C. Davis, Cosmological Evolution on Self-Tuned Branes and the Cosmological Constant, JHEP 0105 (2001) 008, hep-th/0104023.

[10] P. Brax, C. van de Bruck, and A.-C. Davis, Brane-World Cosmology, Bulk Scalars and Perturbations, JHEP 0110 (2001) 026, hep-th/0108215.

[11] P. Brax and A.-C. Davis, On Brane Cosmology and Naked Singularities, Phys. Lett. B513 (2001) 156-162, hep-th/0105269. 
[12] P. Brax, C. van de Bruck, A.-C. Davis, and C. S. Rhodes, Wave function of the radion with bulk scalar field, to appear in Phys. Lett. B., hep-th/0201191.

[13] D. Langlois and M. Rodriguez-Martinez, Brane cosmology with a bulk scalar field, Phys. Rev. D64 (2001) 123507, hep-th/0106245.

[14] A. Mennim and R. Battye, Cosmological expansion on a dilatonic brane-world, Class. Quant. Grav. 18 (2001) 2171-2194, hep-th/0008192.

[15] K. Maeda and D. Wands, Dilaton-gravity on the brane, Phys. Rev. D62 (2000) 124009, hep-th/0008188.

[16] A. Feinstein, K. E. Kunze, and M. A. Vazquez-Mozo, Curved dilatonic brane worlds, Phys. Rev. D64 (2001) 084015, hep-th/0105182.

[17] E. E. Flanagan, S.-H. H. Tye, and I. Wasserman, Brane World Models With Bulk Scalar Fields, Phys. Lett. (2001) 155, hep-th/0110070.

[18] S. C. Davis, Brane Cosmology Solutions with Bulk Scalar Fields, hep-ph/0111351.

[19] C. Csáki, J. Erlich, C. Grojean, and T. J. Hollowood, General Properties of the Self-tuning Domain Wall Approach to the Cosmological Constant Problem, Nucl. Phys. B584 (2000) 359-386, hep-th/0004133.

[20] J. Cline, J. Vinet, Order $\rho^{2}$ Corrections to Randall-Sundrum I Cosmology, gr-qc/0201041.

[21] Y. Himemoto, T. Tanaka, and M. Sasaki, A bulk scalar in the braneworld can mimic the $4 d$ inflaton dynamics, gr-qc/0112027.

[22] S. Mukohyama and L. Kofman, Brane Gravity at Low Energy, hep-th/0112115.

[23] T. Chiba, Scalar-Tensor Gravity in Two 3-brane System, Phys. Rev. D62 (2000) 021502, |gr-qc/0001029.

[24] J. Garriga and T. Tanaka, Gravity in the Randall-Sundrum Brane World, Phys. Rev. Lett. 84 (2000) 2778-2781, hep-th/9911055.

[25] T. Damour and K. Nordtvedt, Tensor-scalar cosmological models and their relaxation toward general relativity, Phys. Rev. D48 (1993) 3436. 